\title{
CHARACTERISTICS OF NITROGEN USE EFFICIENCY AND THEIR MUTUAL RELATIONSHIP IN WINTER WHEAT CULTIVARS
}

\author{
MARTIN UŽÍK, ALŽBETA ŽOFAJOVÁ* \\ Plant Production Research Center Piešt’any
}

UŽÍK, M. - ŽOFAJOVÁ, A.: Characteristic of nitrogen use efficiency and their mutual relationship in winter wheat cultivars. Agriculture (Pol’nohospodárstvo), vol. 59, 2013, no. 4, pp. 149-160.

Field experiments with 12 winter wheat cultivars, which differed in $\mathrm{N}$ grain concentration, were established with three variants of $\mathrm{N}$ fertilisation $(\mathrm{N} 0=0 \mathrm{~kg} \mathrm{~N} / \mathrm{ha}$, $\mathrm{N} 1=100 \mathrm{~kg} \mathrm{~N} / \mathrm{ha}, \mathrm{N} 2=200 \mathrm{~kg} \mathrm{~N} / \mathrm{ha}$ ) in the vegetative years 2004/05 and 2005/06 in Piestany (Borovce). The aims of experiment were to find out the effect of $\mathrm{N}$ applied $(\mathrm{Nf})$ and available $\mathrm{N}(\mathrm{Nfs})$ on differences in $\mathrm{N}$ uptake and $\mathrm{N}$ utilisation. From the primary data of

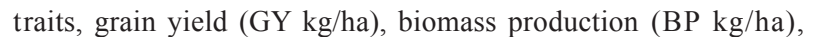
and $\mathrm{N}$ concentration in grain $(\mathrm{Ng})$ and in straw $(\mathrm{Ns})$, other characteristics (NUP, NUE, NUTE) for available and applied $\mathrm{N}$ (Nfs, $\mathrm{Nf}$, respectively) were calculated. In available $\mathrm{N}$, significant differences were seen among cultivars in all characteristics but in applied $\mathrm{N}$ differences in NUP and NUTE among cultivars were not significant. Genetic correlations among traits and characteristics were modified by $\mathrm{N}$ environment. Grain $\mathrm{N}$ concentration $(\mathrm{Ng})$ was, at the low $\mathrm{N}$ rate, in a negative correlation with NUE $\left(r=-0.683^{+}\right)$and in a strong negative correlation with NUTE $\left(r=-0.956^{++}\right)$. At a high $\mathrm{N}$ rate, these correlations had the same character, but were weaker and not significant $(r=-0.560$ and $r=0.570)$. Grain $\mathrm{N}$ concentration $(\mathrm{Ng})$ was more determined by efficiency of $\mathrm{N}$ translocation than by quantity of $\mathrm{N}$ uptake. Selection on the higher NUPE and NUE will be limited by negative correlation between $\mathrm{BP}$ and $\mathrm{N}$ biomass concentration and by low genetic variability of all characteristics of $\mathrm{N}$ uptake and utilisation.

Key words: winter wheat, cultivar, nitrogen use efficiency, nitrogen uptake efficiency, nitrogen utilisation efficiency, available nitrogen

Productive dwarf cultivars and higher doses of nutrients, particularly nitrogen, participated in the increase of cereals yields in the second half of the last century (Austin et al. 1980; Uzik \& Zofajova 2007). In the following decades, it is necessary to increase crop productivity to provide food for a growing population in conditions of decreasing arable land and use of biomass for the production of biofuels. Increasing the actual low efficiency of application of $\mathrm{N}$ fertilisers is an important goal of the research.

Plants are able to accumulate in the grain only $30 \%-40 \%$ of applied nitrogen from fertilisers (Raun \& Johnson 1999) as a large proportion of N penetrates into groundwater and air. Nitrogen use effi- ciency (NUE) is generally defined as grain yield obtained per the unit of applied or available nitrogen in soil. Moll et al. (1982) defined NUE as the product of nitrogen uptake efficiency (NUPE) and nitrogen utilisation efficiency (NUTE), where NUPE [\%] is defined as all $\mathrm{N}$ in the above biomass at maturity divided by sum of the fertiliser $\mathrm{N}$ and soil $\mathrm{N}$ ( $\mathrm{N}$ available) and NUTE defined as ratio of grain yield (in $\mathrm{kg}$ ) to total $\mathrm{N}$ uptake in biomass (NUP in $\mathrm{kg}$ ).

Increasing NUE can be achieved by improving NUPE or NUTE or by increasing the efficiency of both the components. During the past decade, numerous studies have been undertaken for insights into whether NUPE or NUTE plays a more important role in NUE under different $\mathrm{N}$ environments, 
but data in the literature are not unambiguous. Abeledo et al. (2008) report that both $\mathrm{N}$ conversion and $\mathrm{N}$ capture have played a role in the improvement of NUE. According to other authors (LeGouis et al. 2002; Wang et al. 2011), $\mathrm{N}$ uptake in biomass was the most important factor in NUE determination regardless of $\mathrm{N}$ level. Contrary results were obtained by Gaju et al. (2011) who found that the NUPE effect explained only a small amount of phenotypic variance in NUE amongst cultivars, but NUTE affected it up to $61 \%$ and $77 \%$ under high nitrogen (HN) and low nitrogen (LN) conditions, respectively.

In our previous research (Uzik \& Zofajova 2009), we analysed the grain yield structure of 12 winter wheat cultivars, which represent the use in the wider Central European region. In the next work with the same set of cultivars (Uzik \& Zofajova $2012 b$ ), we analysed the dynamics of $\mathrm{N}$ during grain filling. The aim of the present work was to detect, in the same cultivar set, the effect of genotype and $\mathrm{N}$ fertilisation on the differences in total $\mathrm{N}$ uptake and its utilisation for grain yield and biomass and for $\mathrm{N}$ accumulation in grain. Other objectives were to separate the effects of available $\mathrm{N}$ from applied $\mathrm{N}$ on the efficiency of $\mathrm{N}$ uptake and its utilisation and to find out the effect of $\mathrm{N}$ environment and genotype on the relationships among traits of biomass production and $\mathrm{N}$ accumulation and characteristics of the efficiency of $\mathrm{N}$ uptake and $\mathrm{N}$ utilisation.

\section{MATERIAL AND METHODS}

Detailed description of methods for the establishment and management of the experiment was published in the previous papers (Uzik \& Zofajova 2009,2012 b). Field experiments with 12 winter wheat cultivars were established in three variants of $\mathrm{N}$ fertilisation $(\mathrm{N} 0=0 \mathrm{~kg} \mathrm{~N} / \mathrm{ha}, \mathrm{N} 1=100 \mathrm{~kg} \mathrm{~N} / \mathrm{ha}$, $\mathrm{N} 2=200 \mathrm{~kg} \mathrm{~N} / \mathrm{ha}$ ) and in three replications in the vegetation 2004/05 and 2005/06 in Borovce. Pea was the preceding crop.

Nutrition content in soil was the same in both years $(\mathrm{N} 0.098 \%$, humus content $2.084 \%$, pH 7.42, P $59.68 \mathrm{mg} / \mathrm{kg}, \mathrm{K} 183.7 \mathrm{mg} / \mathrm{kg}, \mathrm{Mg} 236.6 \mathrm{mg} / \mathrm{kg}$, Ca $4910 \mathrm{mg} / \mathrm{kg}, \mathrm{NO}_{3} \mathrm{~N} 14.2 \mathrm{mg} / \mathrm{kg}$ and $\mathrm{NH}_{4} \mathrm{~N} 1.942 \mathrm{mg} / \mathrm{kg}$ ). $\mathrm{P}$ and $\mathrm{K}$ fertilisers in the form of superphosphate $\left(26 \% \mathrm{P}_{2} \mathrm{O}_{5}\right)$ and potassium nitrate $\left(60 \% \mathrm{~K}_{2} \mathrm{O}\right)$ at the rates of 50 and $200 \mathrm{~kg} / \mathrm{ha}$, respectively, were applied before sowing. $\mathrm{N}$ fertilisers were applied in the divided rates, at $\mathrm{N} 1$ in the ratio $40: 30: 30 \mathrm{~kg} \mathrm{~N} / \mathrm{ha}$ and at $\mathrm{N} 2$ in the ratio $100: 70: 30 \mathrm{~kg} \mathrm{~N} / \mathrm{ha}$, on three dates. The first date of regenerative $\mathrm{N}$ fertilisation was in the middle of March, the second date in the middle of April and the third date at the end of the first decade of May. $\mathrm{N}$ was applied in the form of ammonium calcite nitrate $(27.5 \% \mathrm{~N})$ in the first and in the second dates and in the form of calcite nitrate $(15.5 \%)$ in the third date in both years.

The evaluated set consisted of seven foreign cultivars (1 - Evropa 90, released in 1990; 2 - Nevesinjka, 1990; 3 - Pobeda, 1990; 4 - Zlatka, 1996; 5 - Sonata from Serbia, 2000; 6 - Renan from France, 1989; 7 - Tamaro from Switzerland, 1992) and of five local cultivars (8 - Ilona, 1989; 9 - Malyska, 2001; 10 - Vanda, 2001; 11 - Petrana, 2002; 12 - Axis, 2003). Grain yield (GY, kg/ha) was assessed from each parcel and from the row $0.5 \mathrm{~m}$ of stand, a plant sample was collected for determination of harvest index (HI). $\mathrm{N}$ concentration in grain $(\mathrm{Ng}, \%)$ and in straw (Ns, \%) were determined by Dumas method using CNS-2000 analyser.

From the primary data of traits, additional characteristics were derived:

- $\mathrm{Ns}[\mathrm{kg} / \mathrm{ha}$ - amount of soil $\mathrm{N}$ was defined as $\mathrm{N}$ amount taken by parcel with maximal $\mathrm{N}$ uptake $\left(\mathrm{ijk} l_{\max }\right)$ on non-fertilised variant N0 (k) in each year (i) and replication (j) (twice it was cultivar (1) Tamaro and once Vanda),

- $\mathrm{Nfs}[\mathrm{kg} / \mathrm{ha}]$ - available $\mathrm{N}$ was expressed as sum of $\mathrm{N}$ quantity in fertilisers ( $\mathrm{Nf}$ in $\mathrm{kg} / \mathrm{ha}$ ) and soil (Ns),

- $\quad \mathrm{BP}[\mathrm{kg} / \mathrm{ha}]$ - biomass production was calculated from grain yield and harvest index (GY/HI),

- $\mathrm{Nb}[\%]$ - concentration $\mathrm{N}$ in biomass at maturity (weighed average from $\mathrm{Nv}$ a $\mathrm{Ng}$ ),

- NUP $[\mathrm{kg} / \mathrm{ha}]-\mathrm{N}$ uptake in biomass,

- NUPE [\%] - efficiency of N uptake (NUP / Nfs) $\times 100$

- $\mathrm{NAg}[\mathrm{kg} / \mathrm{ha}]-\mathrm{N}$ accumulation in grain,

- NAgE [\%] - efficiency of $\mathrm{N}$ accumulation in grain $(\mathrm{NAg} / \mathrm{Nfs}) \times 100$,

- NAs $[\mathrm{kg} / \mathrm{ha}]-$ accumulation $\mathrm{N}$ in straw, 
- NAsE [\%] - efficiency of $\mathrm{N}$ accumulation in straw (NAs / Nfs) $\times 100$,

- NUEg $[\mathrm{kg} / \mathrm{kg}]-\mathrm{N}$ (available) use efficiency $(\mathrm{Nfs})$ on grain yield (GY / Nfs),

- NUEb $[\mathrm{kg} / \mathrm{kg}]-\mathrm{N}$ (available) use efficiency (Nfs) on biomass production (BP / Nfs),

- NUTE $[\mathrm{kg} / \mathrm{kg}]-\mathrm{N}$ utilisation efficiency (GY / NUP). Characteristics for estimation of the effect of applied nitrogen (Nf) were determined as difference between the value of trait in non-fertilised variant and in fertilised one, which was calculated for each year (i), replication (j), $\mathrm{N}$ fertilisation ( $\mathrm{k}$ ) and cultivar (1). This manner of estimation has taken into account differences of mineralisation in the soil microenvironment and specific differences in $\mathrm{N}$ uptake among cultivars:

$$
\mathrm{xf}_{\mathrm{ijk} \mathrm{l}}=\mathrm{xfs}_{\mathrm{ijk} \mathrm{k}}(\mathrm{k}=\mathrm{N} 1 \text { or } \mathrm{N} 2)-\mathrm{x}_{\mathrm{ijkl}}(\mathrm{k}=\mathrm{N} 0) \text {, }
$$

where $\mathrm{x}$ can be GY, BP or NUP.

- $\quad$ NUPf $=$ NUPfs - NUPs $-\mathrm{N}$ uptake of applied $\mathrm{N}$ fertiliser,

- NUPfE [\%] $=(\mathrm{NUPf} / \mathrm{Nf}) \times 100-$ efficiency of $\mathrm{N}$ uptake of applied $\mathrm{N}$ fertiliser,

- $\mathrm{NAgf}[\mathrm{kg} / \mathrm{ha}]=(\mathrm{NAgfs}-\mathrm{NAgNs})-$ accumulation of applied $\mathrm{Nf}$ in grain,

- $\mathrm{NAgfE}[\%]=(\mathrm{NAgf} / \mathrm{Nf}) \times 100-$ efficiency of accumulation of applied $\mathrm{Nf}$ in grain,

- $\quad \mathrm{NAsf}[\mathrm{kg} / \mathrm{ha}]=(\mathrm{NAsfs}-\mathrm{NAsNs})-$ accumulation of applied $\mathrm{Nf}$ in grain,

- $\mathrm{NAsfE}[\%]=(\mathrm{NAsf} / \mathrm{Nf}) \times 100-$ efficiency of accumulation of applied $\mathrm{Nf}$ in grain,

- $\quad \mathrm{NUEgf}[\mathrm{kg} / \mathrm{kg}]=(\mathrm{GYf} / \mathrm{Nf})-$ use efficiency of applied $\mathrm{N}$ on grain yield, where $\mathrm{GYf}=\mathrm{GYfs}-$ GYs,

- $\quad \mathrm{NUEbf}=(\mathrm{BPf} / \mathrm{Nf})-$ use efficiency of applied $\mathrm{N}$ on biomass production, where $\mathrm{BPf}=\mathrm{BPfs}-$ BPs,

- NUTEgf $[\mathrm{kg} / \mathrm{kg}]=(\mathrm{GYf} / \mathrm{NUPf})-$ utilisation efficiency of applied $\mathrm{N}$ for grain yield.

Statistical analyses were performed by the software package Statgrafics plus for Windows. The combined ANOVA (year, $\mathrm{N}$ fertilisation, genotype and replication) of the data set and characteristics from all experiments were performed (not shown). Genotypic (cultivar) correlations were calculated from average values for cultivars over years, replications and $\mathrm{N} 0$ variant of fertilisation (rGLN, low $\mathrm{N}$ rate); from average of $\mathrm{N} 1$ and $\mathrm{N} 2$ variants of fertilisation (rGHN, high $\mathrm{N}$ rate); and from average characteristics N0, N1 and N2 for rGF. By combinations of factors - year, $\mathrm{N}$ fertilisation, replication $(2 \times 3 \times 2=12)-12$ environments differentiated by $\mathrm{N}$ level were formed, termed as $\mathrm{N}$ environments. $\mathrm{N}$ environments correlations ( $\mathrm{rE}$ ) were calculated from average data of 12 cultivars (hypothetic average cultivar) from each environment.

\section{RESULTS}

Nitrogen uptake (NUP $\mathrm{kg} / \mathrm{ha}$ ) and nitrogen uptake efficiency (NUPE \%) of available nitrogen (NfS) (Table 1)

From the total average amount of available N, $320 \mathrm{~kg} \mathrm{~N} / \mathrm{ha}, 224.4 \mathrm{~kg} \mathrm{~N} / \mathrm{ha}$ (NUP) was taken up, i.e. $72.5 \%$ efficiency of $\mathrm{N}$ uptake (NUPE), by the crop. Characteristics of $\mathrm{N}$ uptake had low experimental error $(\mathrm{Ve})$ and low genetic variability $(\mathrm{Vg})$.

Significant differences were among cultivars in NUP and NUPE. From the other cultivars, only two were different by low NUP and NUPE - Nevesinj$\mathrm{ka}(209.1 \mathrm{~kg} \mathrm{~N} / \mathrm{ha}$ and $66.5 \%)$ and Zlatka $(199.6 \mathrm{~kg}$ $\mathrm{N} / \mathrm{ha}$ and $63.3 \%$ ). From the total amount of available $\mathrm{N}(\mathrm{Nfs})$, only $169.8 \mathrm{~kg} / \mathrm{ha}(\mathrm{NAg})$, i.e. $55.2 \%$ (NAgE), was distributed in the grain. Cultivars Pobeda (58.9\%), Renan (59.2\%) and Axis (58.4\%) exhibited a high portion of $\mathrm{N}$ accumulation in the grain. Cultivar Zlatka accumulated a lower N portion in grain $(50.2 \%)$ than cultivars Tamaro, Malyska and Vanda, which accumulated more than $20 \%$ in the straw (NAsE).

Nitrogen uptake (NUP $\mathrm{kg} / \mathrm{ha}$ ) and nitrogen uptake efficiency (NUPE \%) of applied nitrogen (Nf) (Table 2)

Characteristics of efficiency of applied $\mathrm{N}$ rates estimated as difference between $\mathrm{N}$ fertilised and non-fertilised variant had a high coefficient of experimental error $(\mathrm{Ve})$, which was not caused by high absolute value of MSe, but low difference among $\mathrm{Nx}$ - N0 variant, in which Ve was expressed. Differences among cultivars in the total uptake of applied N (NUPf) were not significant, but effects of year, $\mathrm{N}$ fertilisation (not shown) and cultivar were 
T $\begin{array}{lllllllll} & \text { a } & \mathrm{b} & 1 & \mathrm{e} & 1\end{array}$

$\mathrm{N}$ uptake, $\mathrm{N}$ uptake efficiency, $\mathrm{N}$ accumulation efficiency in grain and in straw of winter wheat cultivars at available $\mathrm{N}$

\begin{tabular}{|c|c|c|c|c|c|c|}
\hline Cultivar & $\begin{array}{c}\text { NUP } \\
{[\mathrm{kg} / \mathrm{ha}]}\end{array}$ & $\begin{array}{c}\text { NUPE } \\
{[\%]}\end{array}$ & $\begin{array}{c}\mathrm{NAg} \\
{[\mathrm{kg} / \mathrm{ha}]}\end{array}$ & $\begin{array}{l}\text { NAgE } \\
{[\%]}\end{array}$ & $\begin{array}{c}\text { NAs } \\
{[\mathrm{kg} / \mathrm{ha}]}\end{array}$ & $\begin{array}{c}\text { NAsE } \\
{[\%]}\end{array}$ \\
\hline Evropa 90 & $222.4^{\mathrm{bcd}}$ & $71.6^{\mathrm{bc}}$ & $171.3^{\text {bcde }}$ & $55.7^{\text {bcde }}$ & $51.0^{\mathrm{ab}}$ & $15.8^{\mathrm{ab}}$ \\
\hline Nevesinjka & $209.1^{\mathrm{ab}}$ & $66.5^{\mathrm{ab}}$ & $163.3^{\mathrm{ab}}$ & $52.2^{\mathrm{ab}}$ & $45.8^{\mathrm{ab}}$ & $14.3^{\mathrm{ab}}$ \\
\hline Pobeda & $238.4^{\mathrm{d}}$ & $75.9^{\mathrm{c}}$ & $184.0^{\mathrm{e}}$ & $58.9^{\mathrm{e}}$ & $54.4^{\mathrm{bc}}$ & $17.1^{\mathrm{bc}}$ \\
\hline Zlatka & $199.6^{\mathrm{a}}$ & $63.3^{\mathrm{a}}$ & $157.5^{\mathrm{a}}$ & $50.2^{\mathrm{a}}$ & $42.2^{\mathrm{a}}$ & $13.1^{\mathrm{a}}$ \\
\hline Sonata & $217.2^{\mathrm{abc}}$ & $70.7^{\mathrm{bc}}$ & $166.6^{\mathrm{bcde}}$ & $54.8^{\mathrm{abcde}}$ & $50.6^{\mathrm{ab}}$ & $15.9^{\mathrm{ab}}$ \\
\hline Renan & $232.6^{\mathrm{cd}}$ & $76.1^{\mathrm{c}}$ & $179.4^{\mathrm{e}}$ & $59.2^{\mathrm{e}}$ & $53.1^{\mathrm{bc}}$ & $16.9^{\mathrm{bc}}$ \\
\hline Tamaro & $232.8^{\mathrm{cd}}$ & $76.7^{\mathrm{c}}$ & $170.9^{\text {bcde }}$ & $56.5^{\text {bcde }}$ & $61.9^{\text {cde }}$ & $20.1^{\text {cde }}$ \\
\hline Ilona & $224.0^{\mathrm{bcd}}$ & $72.0^{\mathrm{bc}}$ & $177.1^{\text {cde }}$ & $56.9^{\text {cde }}$ & $46.9^{\mathrm{ab}}$ & $15.1^{\mathrm{ab}}$ \\
\hline Malyska & $232.8^{\mathrm{cd}}$ & $76.1^{\mathrm{c}}$ & $159.6^{\mathrm{abc}}$ & $53.1^{\mathrm{abc}}$ & $73.2^{\mathrm{e}}$ & $23.0^{\mathrm{e}}$ \\
\hline Vanda & $229.2^{\mathrm{cd}}$ & $75.0^{\mathrm{c}}$ & $163.3^{\mathrm{abcd}}$ & $53.8^{\mathrm{abcd}}$ & $65.9^{\mathrm{de}}$ & $21.2^{\mathrm{de}}$ \\
\hline Petrana & $226.0^{\mathrm{bcd}}$ & $72.3^{\mathrm{bc}}$ & $164.1^{\mathrm{abc}}$ & $52.8^{\mathrm{abc}}$ & $61.9^{\mathrm{cd}}$ & $19.5^{\mathrm{cd}}$ \\
\hline Axis & $228.3^{\mathrm{bcd}}$ & $73.6^{c}$ & $180.1^{\mathrm{de}}$ & $58.4^{\mathrm{de}}$ & $48.2^{\mathrm{ab}}$ & $15.3^{\mathrm{ab}}$ \\
\hline $\bar{x}$ & 224.4 & 72.5 & 169.8 & 55.2 & 54.6 & 17.3 \\
\hline$L S D_{0.05}$ & 20.0 & 6.7 & 12.2 & 4.5 & 11.3 & 3.3 \\
\hline$V g[\%]$ & 4.9 & 5.7 & 5.1 & 5.2 & 16.9 & 17.5 \\
\hline $\mathrm{Ve}[\%]$ & 12.3 & 12.0 & 8.5 & 8.8 & 17.8 & 15.7 \\
\hline
\end{tabular}

Means within columns with different letters are significantly different at $\alpha=0.05$

NUP - N uptake in biomass, NUPE - efficiency of N uptake, NAg - N accumulation in grain, NAgE - efficiency of $\mathrm{N}$ accumulation in grain, NAs - accumulation $\mathrm{N}$ in straw, NAsE - efficiency of $\mathrm{N}$ accumulation in straw

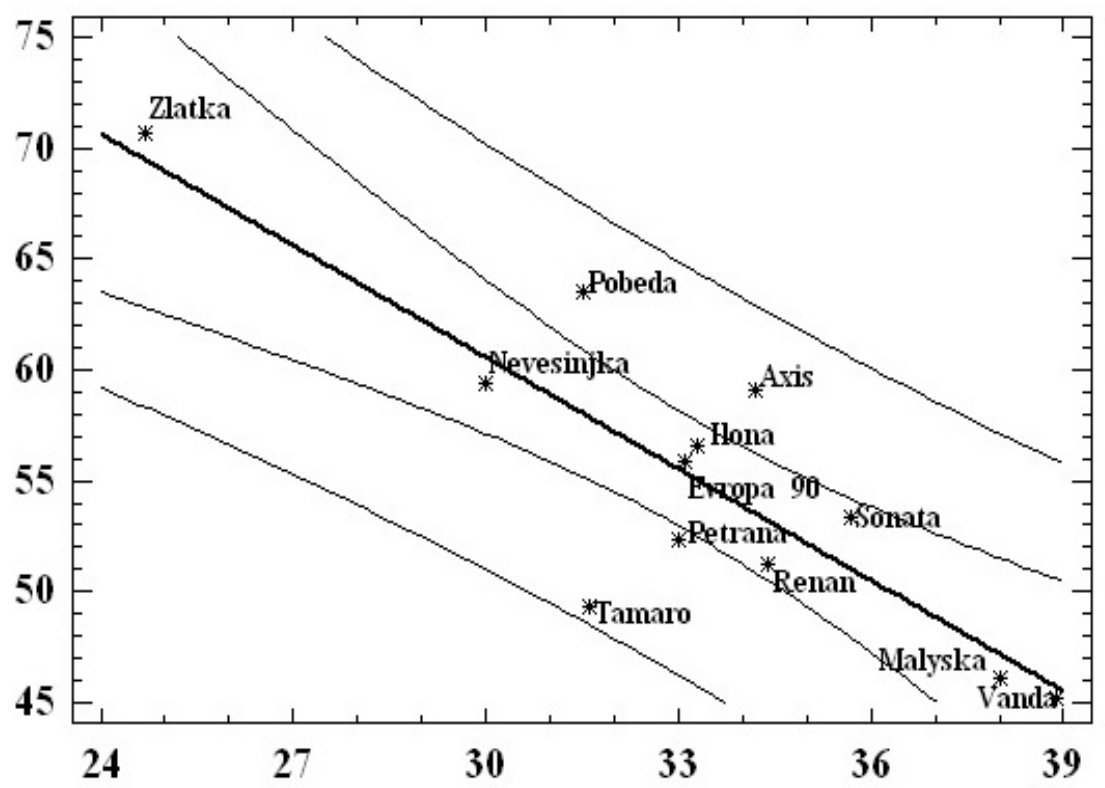

Figure 1. Relationship between NUEg at variant N0 (x axis) and NUEg [\%] at variant N2 (y axis) $(100 \%=$ NUEg at variant N0) 
significant for distribution of applied $\mathrm{Nf}$ in the grain (NAgf) and in the straw (NAsf). Interaction $\mathrm{N}$ fertilisation $\times$ cultivar was not significant (not shown). From the average rate of applied $\mathrm{N}(\mathrm{Nf}=150 \mathrm{~kg} / \mathrm{ha})$ by the cultivar only $65.1 \mathrm{~kg} \mathrm{~N} / \mathrm{ha}$, i.e. $46.9 \%$ (NUPfE), was taken up. Differences among cultivars fluctuated up to the double value of the minimal difference (from $46.2 \mathrm{~kg} / \mathrm{ha}$ Renan to $92.3 \mathrm{~kg} / \mathrm{ha}$ Pobeda). Significant differences were among cultivars in $\mathrm{N}$ distribution in grain, namely in the amount (NAgf) and in efficiency (NAgfE), although experimental error was also high. On average, only $31.9 \mathrm{~kg} / \mathrm{ha}$ or $24.0 \%$ from $150 \mathrm{~kg}$ of applied $\mathrm{N}$ was accumulated in grain.

High $\mathrm{N}$ grain accumulation showed cultivars Pobeda (40.6\%), Ilona (36.0\%) or Axis (34.9\%) and low one cultivar Malyska (7.5\%), in which high N portion accumulated in straw (35.1\%). Portion of $\mathrm{N}$ accumulated in grain and in straw from applied $\mathrm{N}$ was nearly the same $(24.0 \%$ and $22.9 \%)$, while from available $\mathrm{N}(\mathrm{Nfs})$ three times more was distributed in grain than in the straw $(55.2 \%$ vs. $17.3 \%)$ (Table $1)$.

Nitrogen use (NUE $\mathrm{kg} / \mathrm{kg}$ ) and nitrogen utilisation efficiency (NUTE $\mathrm{kg} / \mathrm{kg}$ ) of available nitrogen (Nfs) (Table 3)

On average, $25.4 \mathrm{~kg}$ of grain devolved on $1 \mathrm{~kg}$ of available N. High NUEg values had cultivars Vanda (27.8), Axis (26.7), Malyska (27.4) (all low grain quality) and low one Zlatka (21.1) (high grain quality) and Tamaro (23.3) (low HI). Higher available $\mathrm{N}(\mathrm{Nfs})$ amounts significantly made lower NUEg in $\mathrm{N} 2$ variant to $54.6 \%(\mathrm{~N} 0=100 \%)$.

Interaction cultivar $\times \mathrm{N}$ fertiliser can be expressed by relation between absolute value NUEg on N0 variant (x) to relative value NUEg on N2 variant in percentage $(100 \%=$ NUEg on N0 variant)

T $\mathrm{a} \quad \mathrm{b} \quad 1$ e 2

$\mathrm{N}$ uptake and distribution of $\mathrm{N}$ applied at winter wheat cultivars

\begin{tabular}{|c|c|c|c|c|c|c|}
\hline Cultivar & $\begin{array}{l}\text { NUPf } \\
{[\mathrm{kg} / \mathrm{ha}]}\end{array}$ & $\begin{array}{c}\text { NUPfE } \\
{[\%]}\end{array}$ & $\begin{array}{l}\text { NAgf } \\
{[\mathrm{kg} / \mathrm{ha}]}\end{array}$ & $\begin{array}{c}\text { NAgfE } \\
{[\%]}\end{array}$ & $\begin{array}{c}\text { NAsf } \\
{[\mathrm{kg} / \mathrm{ha}]}\end{array}$ & $\begin{array}{c}\text { NAsfE } \\
{[\%]}\end{array}$ \\
\hline Evropa 90 & 54.8 & 38.3 & $23.5^{\mathrm{ab}}$ & $16.0^{\mathrm{ab}}$ & $31.3^{\mathrm{a}}$ & $22.3^{\mathrm{a}}$ \\
\hline Nevesinjka & 78.1 & 59.1 & $44.9^{\mathrm{cd}}$ & $33.8^{\mathrm{cd}}$ & $33.2^{\mathrm{a}}$ & $25.3^{\mathrm{ab}}$ \\
\hline Pobeda & 92.3 & 65.7 & $56.3^{\mathrm{d}}$ & $40.6^{\mathrm{d}}$ & $36.0^{\mathrm{ab}}$ & $25.0^{\mathrm{ab}}$ \\
\hline Zlatka & 75.2 & 50.3 & $47.2^{\mathrm{cd}}$ & $32.7^{\mathrm{cd}}$ & $28.0^{\mathrm{ab}}$ & $17.6^{\mathrm{abc}}$ \\
\hline Sonata & 55.6 & 40.7 & $23.7^{\mathrm{ab}}$ & $18.5^{\mathrm{abc}}$ & $31.9^{\mathrm{abc}}$ & $22.1^{\mathrm{abc}}$ \\
\hline Renan & 46.2 & 33.0 & $16.8^{\mathrm{a}}$ & $12.6^{\mathrm{a}}$ & $29.3^{\mathrm{abc}}$ & $20.4^{\mathrm{abc}}$ \\
\hline Tamaro & 48.3 & 36.7 & $14.9^{\mathrm{a}}$ & $12.2^{\mathrm{a}}$ & $33.3^{\mathrm{abc}}$ & $24.5^{\mathrm{abc}}$ \\
\hline Ilona & 67.0 & 48.3 & $46.6^{\mathrm{cd}}$ & $36.0^{\mathrm{d}}$ & $20.3^{\mathrm{abc}}$ & $12.3^{\mathrm{abc}}$ \\
\hline Malyska & 58.3 & 42.6 & $7.9^{\mathrm{a}}$ & $7.5^{\mathrm{a}}$ & $50.2^{\mathrm{abc}}$ & $35.1^{\mathrm{abc}}$ \\
\hline Vanda & 49.5 & 35.6 & $14.8^{\mathrm{a}}$ & $12.7^{\mathrm{a}}$ & $34.6^{\mathrm{abc}}$ & $22.9^{\mathrm{abc}}$ \\
\hline Petrana & 83.4 & 62.0 & $38.5^{\mathrm{bc}}$ & $30.6^{\text {bcd }}$ & $44.9^{\mathrm{bc}}$ & $31.4^{\mathrm{bc}}$ \\
\hline Axis & 72.7 & 50.8 & $47.8^{\text {cd }}$ & $34.9^{\mathrm{d}}$ & $24.7^{\mathrm{b}}$ & $16.0^{\mathrm{b}}$ \\
\hline $\bar{x}$ & 65.1 & 46.9 & 31.9 & 24.0 & 33.2 & 22.9 \\
\hline$L S D_{0.05}$ & NS & NS & 78.0 & 15.5 & 19.9 & 15.0 \\
\hline$V g[\%]$ & 23.3 & 23.2 & 51.9 & 48.9 & 16.0 & 13.8 \\
\hline $\mathrm{Ve}[\%]$ & 63.4 & 67.3 & 78.0 & 58.1 & 24.3 & 25.9 \\
\hline
\end{tabular}

Means within columns with different letters are significantly different at $\alpha=0.05$

NUPf - N uptake of applied $\mathrm{N}$ fertiliser, NUPfE - efficiency of $\mathrm{N}$ uptake of applied $\mathrm{N}$ fertiliser, NAgf - accumulation of applied Nf in grain, NAgfE - efficiency of accumulation of applied Nf in grain, NAsf - accumulation of applied Nf in grain, NAsfE - efficiency of accumulation of applied Nf in grain 
(y). Assuming that interaction was not found, relative values should be equal for each cultivar and among parameters $\mathrm{x}, \mathrm{y}, r=0$. However, between NUEg absolute value in N0 variant and the relative on $\mathrm{N} 2$ there was a strong negative correlation $\left(r=-0.851^{++}\right)$(Figure 1) while between absolute values in N0 and N2 variant no correlation was found $(r=0.110)$. The cultivars with low NUEg in N0 variant reduced lesser NUEg in N2 variant than cultivars with high NUEg in N0 (compare Zlatka versus Vanda).

Among cultivars, statistically significant differences were for NUE for biomass (NUEb); also, interaction cultivar $\times \mathrm{N}$ fertilisation was significant (not shown). Coefficient NUEb was more than two times higher $(57.9 \mathrm{~kg} / \mathrm{kg})$ than NUEg. NUEg and NUEb in cultivars were combined in different ways: Vanda (high NUEg and NUEb), Zlatka (low NUEg and NUEb) and Nevesinjka (high NUEg and low NUEb). Differences in N (available) utilisation efficiency for grain (NUTE) among cultivars were highly significant. On average, $34.7 \mathrm{~kg}$ grain devolved on $1 \mathrm{~kg}$ of uptake $\mathrm{N}$ with the range from 29.9 in Tamaro to $37.8 \mathrm{~kg}$ grain per $1 \mathrm{~kg} \mathrm{~N}$ in Sonata.

Nitrogen use (NUE $\mathrm{kg} / \mathrm{kg}$ ) and nitrogen utilisation efficiency (NUTE $\mathrm{kg} / \mathrm{kg}$ ) of applied $N$ (Nf) (Table 4)

Cultivars were significantly different in the reaction on applied $\mathrm{N}$ in biomass production increment (BPf) and in grain yield increment (GYf). Cultivars Nevesinjka, Pobeda, Zlatka, Malyska and Petrana positively responded on applied $\mathrm{N}$ by high BPf and cultivars Nevesinjka, Pobeda and Zlatka had the highest GYf. Cultivar Tamaro had only average biomass production increment and unambiguously the lowest increment of grain.

Biological efficiency of applied $\mathrm{N}$ on grain yield (Nf), so the difference between grain yield in fertilised variants minus non-fertilised variant to applied

T $\quad$ a

$\mathrm{N}$ use (NUE) and utilisation (NUTE) efficiency for cultivars at available $\mathrm{N}$ (Nfs)

\begin{tabular}{|c|c|c|c|c|c|}
\hline \multirow{2}{*}{ Cultivar } & \multirow{2}{*}{$\begin{array}{l}\text { NUEg } \\
{[\mathrm{kg} / \mathrm{kg}]}\end{array}$} & \multicolumn{2}{|c|}{ NUE $[\mathrm{kg} / \mathrm{kg}]$} & \multirow{2}{*}{$\begin{array}{l}\mathrm{NUEb} \\
{[\mathrm{kg} / \mathrm{kg}]}\end{array}$} & \multirow{2}{*}{$\begin{array}{l}\text { NUTE } \\
{[\mathrm{kg} / \mathrm{kg}]}\end{array}$} \\
\hline & & No & $\begin{array}{c}\mathrm{N} 2[\%] \\
(\mathrm{N} 0=100 \%)\end{array}$ & & \\
\hline Evropa 90 & $24.9^{\mathrm{bcd}}$ & $33.1^{\mathrm{bcd}}$ & $55.9^{\mathrm{abc}}$ & $55.3^{b}$ & $34.5^{\mathrm{bcd}}$ \\
\hline Nevesinjka & $24.5^{\mathrm{bc}}$ & $30.0^{\mathrm{b}}$ & $59.4^{\mathrm{bcd}}$ & $49.8^{\mathrm{a}}$ & $36.8^{\mathrm{ef}}$ \\
\hline Pobeda & $25.7^{\text {cdef }}$ & $31.5^{\mathrm{bc}}$ & $63.5^{\mathrm{cd}}$ & $58.1^{\mathrm{bc}}$ & $33.7^{\mathrm{bc}}$ \\
\hline Zlatka & $21.1^{\mathrm{a}}$ & $24.7^{\mathrm{a}}$ & $70.7^{\mathrm{d}}$ & $45.4^{\mathrm{a}}$ & $33.4^{\mathrm{bc}}$ \\
\hline Sonata & $26.9^{\mathrm{efg}}$ & $35.7^{\mathrm{def}}$ & $53.4^{\mathrm{abc}}$ & $56.5^{\mathrm{b}}$ & $37.8^{\mathrm{f}}$ \\
\hline Renan & $25.5^{\mathrm{cdef}}$ & $34.4^{\text {cde }}$ & $51.2^{\mathrm{ab}}$ & $58.7^{\mathrm{bcd}}$ & $33.1^{\mathrm{b}}$ \\
\hline Tamaro & $23.3^{b}$ & $31.6^{\mathrm{bcd}}$ & $49.3^{\mathrm{ab}}$ & $62.6^{\mathrm{cd}}$ & $29.9^{\mathrm{a}}$ \\
\hline Ilona & $26.0^{\text {cdefg }}$ & $33.3^{\mathrm{bcd}}$ & $56.6^{\mathrm{abc}}$ & $59.5^{\text {bcd }}$ & $35.8^{\text {de }}$ \\
\hline Malyska & $27.4^{\mathrm{fg}}$ & $38.0^{\text {ef }}$ & $46.1^{\mathrm{a}}$ & $62.4^{\mathrm{cd}}$ & $35.3^{\text {cde }}$ \\
\hline Vanda & $27.8^{\mathrm{g}}$ & $38.9^{f}$ & $45.2^{\mathrm{a}}$ & $64.1^{\mathrm{d}}$ & $36.4^{\mathrm{def}}$ \\
\hline Petrana & $25.3^{\text {cde }}$ & $33.0^{\mathrm{bcd}}$ & $52.3^{\mathrm{abc}}$ & $60.0^{\mathrm{bcd}}$ & $34.6^{\mathrm{bcd}}$ \\
\hline Axis & $26.7^{\text {defg }}$ & $34.2^{\text {cde }}$ & $59.1^{\text {bcd }}$ & $62.2^{\mathrm{cd}}$ & $35.9^{\mathrm{de}}$ \\
\hline $\bar{x}$ & 25.4 & 33.2 & 54.6 & 57.9 & 34.7 \\
\hline$L S D_{0.05}$ & 1.9 & 4.1 & 12.5 & 5.4 & 1.9 \\
\hline$V g[\%]$ & 7.35 & 11.2 & 13.4 & 9.6 & 6.1 \\
\hline $\mathrm{Ve}[\%]$ & 9.21 & 8.7 & 15.9 & 9.2 & 7.5 \\
\hline
\end{tabular}

Means within columns with different letters are significantly different at $\alpha=0.05$

NUEg - N (available) use efficiency (Nfs) on grain yield, NUE - N use efficiency, NUEb - N (available) use efficiency (Nfs) on biomass production, NUTE - N utilisation efficiency 
$\mathrm{N}(\mathrm{NUEgf}=\mathrm{GYf} / \mathrm{Nf})$ was only $4.7 \mathrm{~kg}$ grain per $1 \mathrm{~kg}$ $\mathrm{Nf}$, so five times lower than in available $\mathrm{N}$ (Table $3)$. Significant differences were present among cultivars; some of them produced approximately $10 \mathrm{~kg}$ grains per $1 \mathrm{~kg}$ of applied fertilisers as Nevesinjka (9.9), Pobeda (10.5) and Zlatka (11.0), while cultivars Malyska and Vanda utilised applied $\mathrm{N}$ only for vegetative biomass production and on the contrary their grain yield decreased at higher $\mathrm{N}$ rates (Table 4).

Biological efficiency of applied $\mathrm{N}$ on biomass production was five times lower $(13.0 \mathrm{~kg}$; $\mathrm{BPf} / 1 \mathrm{~kg}$ $\mathrm{Nf}$ ) than available $\mathrm{N}$ and differences among cultivars were statistically significant. Cultivars were significantly different in the ratio NUEgf : NUEbf. Higher biomass production per $1 \mathrm{~kg}$ of applied $\mathrm{N}$ than grain production was seen in cultivars Evropa 90, Nevesinjka, Pobeda, Tamaro and Petrana and in other cultivars biomass production equalled to the grain production, and so biomass increase was only in grain (Zlatka, Sonata, Renan, eventually Axis).

Utilisation efficiency of applied $\mathrm{N}$ for grain (NUTEf) e.g. ratio of the difference of grain yield between fertilised and non-fertilised variant to the difference of amount of $\mathrm{N}$ uptake between fertilised and non-fertilised variants was on average low; only $2.05 \mathrm{~kg}$ of grain and differences among cultivars were not statistically significant due to the high experimental error.

Cultivars can be categorised into several groups according to utilisation of applied $\mathrm{N}$ on grain production. Into the first group belonged cultivars which produced on $1 \mathrm{~kg} \mathrm{~N}$ above average grain yield: Zlatka 28.4, Pobeda 16.8 and Renan $15.2 \mathrm{~kg} / \mathrm{kg}$; into the second group belonged cultivars which accumulated $\mathrm{N}(\mathrm{Nf})$ in vegetative biomass and made lower the grain yield: Malyska - 40.3, Vanda -28.6 and Tamaro $-4.7 \mathrm{~kg} / \mathrm{kg}$. Nearly all cultivars utilised

T a b

$\mathrm{N}$ use (NUE) and $\mathrm{N}$ utilisation (NUTE) efficiency for cultivars at $\mathrm{N}$ applied

\begin{tabular}{|c|c|c|c|c|c|}
\hline Cultivar & GYf $[\mathrm{kg}]$ & $\operatorname{BPf}[\mathrm{kg}]$ & $\begin{array}{l}\text { NUEgf } \\
{[\mathrm{kg} / \mathrm{kg}]}\end{array}$ & $\begin{array}{l}\text { NUEbf } \\
{[\mathrm{kg} / \mathrm{kg}]}\end{array}$ & $\begin{array}{l}\text { NUTEf } \\
{[\mathrm{kg} / \mathrm{kg}]}\end{array}$ \\
\hline Evropa 90 & $340^{\mathrm{cd}}$ & $1950^{\text {cdef }}$ & $4.8^{\text {cde }}$ & $12.9^{\mathrm{abcd}}$ & 5.6 \\
\hline Nevesinjka & $1210^{\mathrm{ef}}$ & $3220^{\text {ef }}$ & $9.9^{\mathrm{fg}}$ & $25.7^{\mathrm{d}}$ & 8.7 \\
\hline Pobeda & $1450^{\text {ef }}$ & $3560^{\mathrm{f}}$ & $10.5^{g}$ & $26.2^{\mathrm{d}}$ & 16.8 \\
\hline Zlatka & $1560^{f}$ & $2800^{\mathrm{def}}$ & $11.0^{\mathrm{g}}$ & $18.7^{\mathrm{cd}}$ & 28.4 \\
\hline Sonata & $350^{\mathrm{cd}}$ & $1530^{\text {cde }}$ & $3.0^{\mathrm{bcd}}$ & $12.1^{\mathrm{abcd}}$ & 5.9 \\
\hline Renan & $120^{\mathrm{bc}}$ & $590^{\mathrm{abc}}$ & $1.4^{\mathrm{abc}}$ & $4.2^{\mathrm{abc}}$ & 15.2 \\
\hline Tamaro & $50^{\mathrm{abc}}$ & $1590^{\text {cde }}$ & $1.1^{\mathrm{abc}}$ & $13.8^{\mathrm{bcd}}$ & -4.7 \\
\hline Ilona & $900^{\text {def }}$ & $-260^{\mathrm{ab}}$ & $7.3^{\mathrm{efg}}$ & $-1.5^{\mathrm{ab}}$ & 8.2 \\
\hline Malyska & $-460^{\mathrm{ab}}$ & $2530^{\text {def }}$ & $-2.1^{\mathrm{a}}$ & $20.9^{\mathrm{cd}}$ & -40.3 \\
\hline Vanda & $-570^{\mathrm{a}}$ & $-720^{\mathrm{a}}$ & $-1.1^{\mathrm{ab}}$ & $-4.8^{\mathrm{cd}}$ & -28.6 \\
\hline Petrana & $380^{\text {cd }}$ & $2540^{\text {def }}$ & $3.9^{\text {cde }}$ & $19.3^{\mathrm{cd}}$ & 2.4 \\
\hline Axis & $870^{\text {de }}$ & $1330^{\mathrm{bcd}}$ & $6.2^{\mathrm{def}}$ & $8.9^{\mathrm{abcd}}$ & 7.0 \\
\hline $\bar{x}$ & 520 & 1720 & 4.7 & 13.0 & 2.05 \\
\hline$L S D_{0.05}$ & 660 & 1770 & 4.1 & 17.8 & NS \\
\hline$V g[\%]$ & 134.7 & 77.4 & 95.1 & 76.4 & 930 \\
\hline$V e[\%]$ & 22.5 & 155 & 47.0 & 92.9 & 34.0 \\
\hline
\end{tabular}

Means within columns with different letters are significantly different at $\alpha=0.05$

GYf - grain yield increment, BPf - biomass production increment, NUEgf - use efficiency of applied $\mathrm{N}$ on grain yield, NUEbf - use efficiency of applied $\mathrm{N}$ on biomass production, NUTEf - utilisation efficiency of applied $\mathrm{N}$ for grain 
applied uptake $\mathrm{N}(\mathrm{Nf})$ for biomass production except cultivars Ilona and Vanda, which made lower biomass production.

Genetic correlations among traits and characteristics on the low and high $\mathrm{N}$ rate (Table 5)

NUEg on low $\mathrm{N}$ rate was in a stronger relationship with NUP $\left(r=0.735^{++}, R^{2}=50 \%\right)$ than with NUTE ( $r=0.548, R^{2}=30 \%$ ); on the contrary, on high $\mathrm{N}$ rate NUEg was in a stronger relationship with NUTE $\left(r=0.656^{+}\right)$than with NUP $(r=0.444$, $R^{2}=20 \%$ ).

The amount of $\mathrm{N}$ uptake (NUP) determined the differences among cultivars in NUEg, on the low $\mathrm{N}$ rate to $50 \%$, but on the high $\mathrm{N}$ rate only to $20 \%$ $(r G x(\mathrm{NUP} \times \mathrm{NUEg})=0.570)$. No correlation was between NUP and its utilisation for grain NUTEg.
Significant differences among cultivars were in NUTEg (29.9 to $37.83 \mathrm{~kg}$ grain per $\mathrm{kg} \mathrm{N}$ ) (Table 3) and were more causally determined by HI than by $\mathrm{N}$ uptake, as HI determined NUTEg on low N rate, circa $36 \%(r=0.569)$ and on the high $\mathrm{N}$ rate, up to $64 \%$ $\left(r=0.809^{++}\right)$.

Positive correlations among some variables were expected as one variable is the component of the other one, for example, NUP $\times \mathrm{NAg}=(\mathrm{NAs}+$ $\mathrm{NAg}) \times \mathrm{NAg}$. Relation NUP $\times$ NAg was modified by $\mathrm{N}$ content rate in the environment. At the low $\mathrm{N}$ rate, NUP determined $\mathrm{N}$ in grain $(\mathrm{NAg})$ was very strong $\left(r=0.964^{++}\right)$and weaker on the high $\mathrm{N}$ rate $(r=0.499)$. At the low $\mathrm{N}$ rate cultivars were not differentiated in efficiency of $\mathrm{N}$ translocation in the grain, but at $\mathrm{N}$ abundance in the environment the amount of $\mathrm{N}$ in grain was more dependent on ef-

T a b 1 e 5

Genetic ( $r G L N$ low $\mathrm{N}$ rate, $r G H N$ high $\mathrm{N}$ rate) and $\mathrm{N}$ environments $(r E)$ correlation among production biomass, grain yield, $\mathrm{N}$ concentration and characteristics of $\mathrm{N}$ use efficiency for grain yield and biomass production

\begin{tabular}{|l|c|c|c|c|c|}
\hline $\begin{array}{l}\text { Combination of } \\
\text { traits }\end{array}$ & $r G L N$ & $r G H N$ & $r G x$ & $r G f$ & $r E$ \\
\hline $\mathrm{NUEg} \times \mathrm{NUP}$ & $0.735^{++}$ & 0.444 & 0.570 & $0.762^{++}$ & $-0.747^{++}$ \\
$\mathrm{NUEg} \times \mathrm{NUTE}$ & 0.548 & $0.656^{+}$ & $0.603^{+}$ & $0.807^{++}$ & $0.924^{++}$ \\
$\mathrm{NUP} \times \mathrm{NUTE}$ & -0.157 & -0.337 & -0.281 & 0.430 & $-0.870^{++}$ \\
$\mathrm{HI} \times \mathrm{NUTE}$ & 0.569 & $0.809^{++}$ & $0.714^{++}$ & $0.644^{+}$ & $0.694^{+}$ \\
$\mathrm{NUP} \times \mathrm{NAg}$ & $0.964^{++}$ & 0.499 & $0.585^{+}$ & $0.873^{++}$ & $0.908^{++}$ \\
$\mathrm{NAs} \times \mathrm{NAg}$ & $0.681^{+}$ & -0.531 & -0.244 & -0.411 & 0.192 \\
$\mathrm{Ng} \times \mathrm{GY}$ & $-0.720^{++}$ & -0.495 & $-0.752^{++}$ & 0.082 & $0.864^{++}$ \\
$\mathrm{Ng} \times \mathrm{NUEg}$ & $-0.683^{+}$ & -0.560 & $-0.758^{++}$ & 0.126 & $-0.696^{+}$ \\
$\mathrm{Ng} \times \mathrm{NUTEg}$ & $-0.956^{++}$ & -0.570 & $-0.796^{++}$ & -0.074 & $-0.754^{++}$ \\
$\mathrm{Ng} \times \mathrm{NUP}$ & -0.028 & 0.082 & -0.039 & 0.498 & $0.961^{++}$ \\
$\mathrm{Ng} \times \mathrm{Ns}$ & -0.337 & $-0.687^{+}$ & $-0.615^{+}$ & 0.166 & 0.119 \\
$\mathrm{NUEb} \times \mathrm{NUP}$ & $0.829^{++}$ & $0.808^{++}$ & $0.866^{++}$ & $0.616^{+}$ & $-0.757^{++}$ \\
$\mathrm{Nb} \times \mathrm{BP}$ & $-0.744^{++}$ & $-0.859^{++}$ & $-0.905^{++}$ & -0.365 & $0.647^{+}$ \\
$\mathrm{Nb} \times \mathrm{NUP}$ & -0.214 & -0.424 & $-0.597^{+}$ & 0.448 & $0.897^{++}$ \\
$\mathrm{Nb} \times \mathrm{NUEb}$ & $-0.719^{++}$ & $-0.867^{++}$ & $-0.900^{++}$ & -0.406 & $-0.928^{++}$ \\
$\mathrm{Nb} \times \mathrm{NUTEb}$ & $-0.998^{++}$ & $-0.998^{++}$ & $-0.991^{++}$ & -0.371 & $-0.996^{++}$ \\
\hline
\end{tabular}

${ }^{+} P<0.05 ;{ }^{++} P<0.01$

NUEg - N (available) use efficiency (Nfs) on grain yield, NUP - N uptake in biomass, NUTE - N utilisation efficiency, HI - harvest index, NAg - N accumulation in grain, NAs - N accumulation in straw, Ng $-\mathrm{N}$ concentration in grain, GY - grain yield, NUEg - N (available) use efficiency (Nfs) on grain yield, NUTEg - N utilisation efficiency for grain, $\mathrm{Ns}-\mathrm{N}$ concentration in straw, NUEb - N (available) use efficiency (Nfs) on biomass production, $\mathrm{Nb}$ - concentration $\mathrm{N}$ in biomass at maturity, $\mathrm{BP}$ - biomass production was calculated from grain yield and harvest index, NUTEb - N utilisation efficiency for biomass 
ficiency of $\mathrm{N}$ translocation than on $\mathrm{N}$ in biomass. This also indicates strong correlation between NAg and NAs at the low $\mathrm{N}$ rate $\left(r=0.681^{+}\right)$, but negative and not a significant one $(r=-0.531)$ at the high $\mathrm{N}$ rate. $\mathrm{N}$ grain concentration $(\mathrm{Ng})$ on the low $\mathrm{N}$ rate was in negative correlation with the grain yield $\left(r=-0.720^{++}\right)$with the use of $\mathrm{N}$ on grain (NUEg) $\left(r=-0.683^{+}\right)$and in negative a very strong correlation with NUTE $\left(r=-0.956^{++}\right)$(Figure 2a). At the high $\mathrm{N}$ rate correlations had the same character, but were weaker and non-significant in the same order $(r=-0.495, r=-0.560$ and $r=-0.570)$ (Figure $2 b)$. $\mathrm{N}$ concentration in grain $(\mathrm{Ng})$ was not at all determined by the amount of $\mathrm{N}$ uptake, but by efficiency of translocation $\mathrm{N}$ from straw, when between $\mathrm{Ng}$ and Ns there was a strong negative correlation $\left(r=-0.687^{+}\right)$on the high $\mathrm{N}$ rate and weaker on the low rate $(r=-0.337)$. Similarly, like between $\mathrm{N}$ grain concentration and grain yield, negative correlations were also between $\mathrm{N}$ biomass concentration and biomass production, however, not modified by $\mathrm{N}$ level in the environment.

$N$ environments correlations among traits and characteristics (Table 5)

Correlations among traits and characteristics conditioned by variability of $\mathrm{N}$ environment $(r E)$ were positive if expressed in absolute values, but if characteristics were expressed in $\mathrm{N}$ units, correlations were negative due to decreasing of efficiency of $\mathrm{N}$ unit with increasing of $\mathrm{N}$ amount in the envi- ronment, e.g. $r E(\mathrm{Ng} \times \mathrm{GY})=+0.864^{++}$but $r E(\mathrm{Ng} \times$ NUTEg) $=-0.754^{++}$.

\section{DISCUSSION}

The difference between total uptake of $224.4 \mathrm{~kg} \mathrm{~N} / \mathrm{ha}$ and applied $150 \mathrm{~kg} \mathrm{~N} / \mathrm{ha}$ must come from mineralisation of soil $\mathrm{N}$ that was necessary to estimate for objective assessment of $\mathrm{N}$ efficiency available $\mathrm{N}$ (Nfs) and $\mathrm{N}$ from applied fertilisers (Nf). Ns amount determined by chemical analyses of soil can be lower than $\mathrm{N}$ uptake, so efficiency of $\mathrm{N}$ uptake is overestimated (Gaju et al. 2011) and vice versa estimation Ns according to non-fertilised variant (Guarda et al. 2004) can be underestimated, as it is limited by average genotypic capacity of $\mathrm{N}$ uptake (sink). The strong correlation between $\mathrm{Nfs}$ and $\mathrm{N}$ biomass concentration $\left(R^{2}=93 \%\right)$ documented reliability of Ns estimation by the plot of non-fertilised variant with maximal $\mathrm{N}$ uptake in each replication and year (Figure 3). NUPE of available Nfs, circa $70 \%$ and eventually applied $\mathrm{N}$ circa $65 \%$ in biomass correspond at $\mathrm{HI}=0.4$ to circa $30 \%$ of apparent $\mathrm{N}$ recovery $\mathrm{N}$ in grain (ARf \%), which is in accordance with the literature data (Ortiz-Monasterio et al. 1997; Raun and Johnson 1999; Guarda et al. 2004).

There are two ways how to improve $\mathrm{N}$ uptake, either better fertiliser management or better crop va-
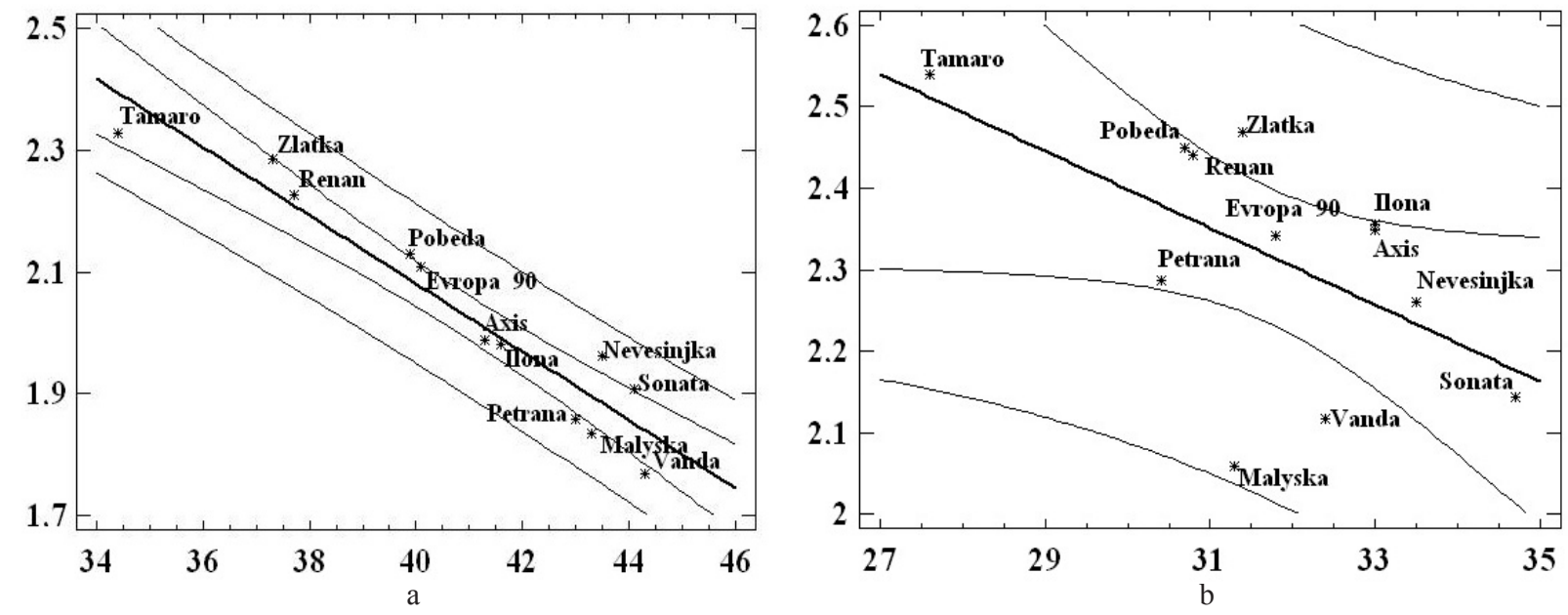

Figure 2. Relationship between NUTE $[\mathrm{kg} / \mathrm{kg}]$ (x axis) and $\mathrm{N}$ concentration in grain $(\mathrm{Ng})[\%]$ (y axis) in low N environment (a) and high $\mathrm{N}$ environment (b) 
rieties. The second way, breeding varieties capable of recovering more $\mathrm{N}$ from the soil and fertilisers, assumes the presence of genetic variability in sink (NUP). Genetic progress in biomass production in one of two basic sink components for NUP was low or none (Austin et al. 1980; Uzik \& Zofajova 2007). In the evaluated cultivar set, low genetic differences were found in NUP in which variability of biomass production contributed positively $\left(r=0.877^{++}\right)$, but $\mathrm{N}$ concentration had a reducing effect $\left(r=-0.597^{+}\right)$. Similarly, Barraclough et al. (2010) found differences in NUP whereby most of the variation was due to differences in biomass rather than to differences in $\mathrm{N}$ concentration.

For increasing efficiency of nitrate intake from the topsoil, the higher root density is generally recommended (Bengough et al. 2006). Wang et al. (2011) obtained genotype KN9204, producing biomass and root dry weight per plant significantly higher than standard on the high $\mathrm{N}$ rate, but had under average $\mathrm{N}$ grain concentration and $\mathrm{N}$ biomass concentration. This is in congruence with the trend of present genetic progress when grain yield was achieved by the redistribution of biomass and not by its raising (Austin et al. 1980; Uzik \& Zofajova 2007). Also the translocation of dry matter of roots has been involved in the genetic progress in grain yield (Uzik \& Zofajova 2007).

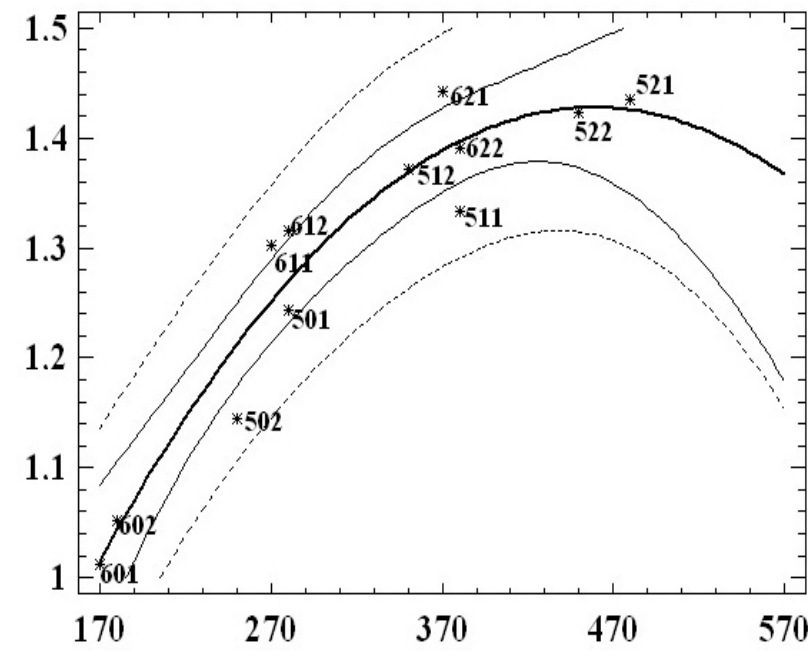

Figure 3. Relationship between amount of available $\mathrm{N}$ in environment ( $\mathrm{Nfs}[\mathrm{kg} / \mathrm{ha}]$ ( $\mathrm{x}$ axis) and $\mathrm{N}$ biomass concentration $(\mathrm{Nb})$ ( $\mathrm{y}$ axis) at environment ijk where $\mathrm{i}=5,6$ year, $\mathrm{j}=0,1$, $2 \mathrm{~N}$ variant, $\mathrm{k}=1,2$
Determination of $\mathrm{N}$ in biomass by $\mathrm{N}$ content in the environment on $93 \%$ indicates that $\mathrm{N}$ resorption by wheat root from the environment is rather passive than active; however, correlation coefficient between $\mathrm{Nfs}$ and $\mathrm{Nb}$ varied among cultivars from $r=0.66$ (Malyska, low grain quality) to $r=0.90$ (Zlatka and Nevesinjka, high grain quality) (not shown). Probably it is possible to select genotypes having the greatest capacity to accumulate an excess of $\mathrm{N}$ during periods of abundant $\mathrm{N}$ supply (Hirel \& Lemaire 2006).

No relation was shown between NUP and NUTE in this experiment as well as in the historical set of cultivars (Baraclough et al. 2010; Uzik \& Zofajova 2012a). The ratio of grain yield to NUP (NUTE) is not determined by NUP but by the HI, in which genetic progress was found (Austin et al. 1980; Uzik \& Zofajova 2007) and which is latent in the formula $(\mathrm{NUTE}=(\mathrm{GY} / \mathrm{BP}) \times \mathrm{Nb}=\mathrm{HI} / \mathrm{Nb})$. In the evaluated set, differences among cultivars in NUTE were determined by $\mathrm{HI}$, on the low $\mathrm{N}$ rate $(30 \%)$ and on the high $\mathrm{N}$ rate (up to $65 \%$ ). Genetic variability of NUTE may be interpreted by HI variability and by $\mathrm{N}$ dilution in biomass also in the set of cultivars historically less differentiated.

We found out that in low $\mathrm{N}$ rate environment, NUE was more determined by NUPE (54\%) than by NUTE (30\%), but in high N rate environment NUTE was more significant than NUPE, which corresponds with the results of several authors (Ortiz-Monasterio et al. 1997; LeGouis et al. 2002) but is not in accord with other authors mentioned in the introduction. Barraclough et al. (2010) observed that NUTE was the key component determining genetic variation in GY under either low or high $\mathrm{N}$ conditions. On the contrary, Wang et al. (2011) found NUPE determines NUE more than NUTE regardless of N conditions. The differently reported results may be due to different variability of tested genotypes in HI and $\mathrm{Nb}$ and $\mathrm{N}$ level in environment which modifies the relationships.

Between grain yield (or NUEg) and N grain concentration, a negative correlation was found out, stronger on the low $\mathrm{N}$ rate than on the high $\mathrm{N}$ rate, which is analogous to other authors (Barraclough et al. 2010). But it is not consistent with the data of Wang et al. (2011) who found out only weak correlation on the low and high $\mathrm{N}$ rate. We have found out 
strong negative correlations between NUTE and Ng, which in the low $\mathrm{N}$ environment was so strong that it can be rightfully marked as functional (Barraclough et al. 2010) and was as strong as in the historical set of genotypes (Baraclough et al. 2010; Uzik \& Zofajova 2012a). In the high $\mathrm{N}$ environment, correlation between NUTE and N grain concentration was also negative, but weak, which indicates that the power of linkage is dependent on environment and has no pleiotropic character.

Correlation between $\mathrm{Ng}$ and NUTE is more easily interpreted when characteristic NUTE is expressed in primary variables from which it was derived $(\mathrm{GY} / \mathrm{BP}) \times(\mathrm{Nv}+\mathrm{Ng})=\mathrm{HI} /(\mathrm{Ns}+\mathrm{Ng})$. As it is seen, genetic correlation between $\mathrm{Ng}$ and grain yield per one unit of $\mathrm{N}$ uptake was modified by selection, namely, by redistribution of dry matter in favour of grain yield (HI) and by efficiency of $\mathrm{N}$ translocation, which altered the ratio straw Ns to grain $\mathrm{Ng}$. Between the year of cultivar release and ratio Ns / Nba ( $\mathrm{N}$ concentration in straw to $\mathrm{N}$ concentration in biomass at anthesis) marked as RNT (relative $\mathrm{N}$ translocation) and ratio $\mathrm{Ng}$ : Ns marked as RNA (relative $\mathrm{N}$ accumulation) was a very strong correlation (Uzik \& Zofajova 2012b). The assumption that negative correlation between NUTE and $\mathrm{Ng}$ is possible to modify by selection was demonstrated on hypothetic populations (Uzik \& Zofajova 2012a).

Characteristics of efficiency of uptake and utilisation of available $\mathrm{N}(\mathrm{Nfs})$ had a low genetic variability and interaction genotype $\times \mathrm{N}(\mathrm{Nfs})$ was not observed. Characteristics of applied $\mathrm{N}$ had a higher genetic variability, namely, in the uptake of applied $\mathrm{N}$ four times and in accumulation in grain (NAgf) ten times higher. The method could be utilised for the detection of genotypes with more effective utilisation of applied N as Guarda et al. (2004) similarly exploited it for estimation of genetic progress for apparent nitrogen recovery (ARf) and in agronomic efficiency of applied $\mathrm{N}(\mathrm{Ae} \%)$.

In accordance with the opinion of other authors (Barraclough et al. 2010), namely, for organic farming (Konvalina et al. 2009), it is necessary to extend genetic variability for characteristics of efficiency of uptake and utilisation of N. Simultaneous selection in the environments at low $\mathrm{N}$ and high $\mathrm{N}$ availability will allow easier detecting of favourable recombinants and separate effects of available and applied N.

\section{CONCLUSIONS}

Among cultivars there were low but significant differences in N uptake (NUP) and in efficiency of $\mathrm{N}$ uptake of available $\mathrm{N}$ (soil Ns and applied Nf), while cultivars were significantly differentiated in the distribution of $\mathrm{N}$ in straw and grain and in the utilisation for grain formation (NUTE).

NUE was about the same proportion determined by NUPE and by NUTE, while no relation was present between them, which means that breeding progress and differentiation among cultivars in grain yield were conditioned by $\mathrm{HI}$ and not by quantity of $\mathrm{N}$ uptake.

The controversial relationship between utilisation of $\mathrm{N}$ uptake on grain yield (NUTE) and $\mathrm{N}$ grain accumulation $(\mathrm{Ng})$ was conditioned by the quantity of $\mathrm{N}$ available in environment. In the environment between NUTE and Ng there was a strong significant negative correlation $\left(r=-0.956^{++}\right)$at low $\mathrm{N}$ rate, which was weaker and non-significant at high $\mathrm{N}$ rate $(r=-0.570)$.

It is necessary to extend genetic variability for traits of efficiency of uptake and utilisation of $\mathrm{N}$. Simultaneous selection in the environments at low $\mathrm{N}$ and high $\mathrm{N}$ availability will allow easier detection of favourable recombinants and evaluation of the effects of available and applied N.

Acknowledgements. This work was supported by the Operational Programme Research and Development for the project: "Implementation of the research of plant genetic resources and its maintaining in the sustainable management of Slovak republic" (ITMS: 26220220097), co-financed through the resources of the European Union Fund for Regional Development.

\section{REFERENCES}

ABEledo, L.G. - CALDERINI, D.F. - SLAFER, G.A. 2008. Nitrogen economy in old and modern malting barleys. In Field Crops Research, vol. 106, pp. 171-178. DOI:10.1016/j.fcr.2007.11.006. 
AUSTIN, R.B. - BINGHAM, J. - BLACKWELL, R.D. EVANS, L.T. - FORD, M.A. MORGAN, C.L. - TAYLOR, M. 1980. Genetic improvements in winter wheat yields since 1900 and associated physiological changes. In Journal of Agriculture Science, vol. 94, pp. 675-689.

BARRACLOUGH, P.B. - HOWARTH, J.R. - JONES, J. - LOPEZ-BELLIDO, R. - PARMAR, S. - SHEPHERD, E.C. - HAWKESFORD, M.J. 2010. Nitrogen efficiency of wheat: Genotypic and environment variation and prospects for improvement. In European Journal of Agronomy, vol. 33, pp. 1-11. http://dx.doi. org/10.1016/j.eja.2010.01.005.

BENGOUGH, A.G. - BRANSBY, M.F. - HANS, J. McKENNA, S.J. - REBERTS, T.J. - VALENTINE, T.A. 2006. Root response to soil physical conditions; growth dynamics from field to cell. In Journal of Experimental Botany, vol. 57, pp. 437-447. DOI: $10.1093 / \mathrm{jxb} / \mathrm{erj} 003$.

GAJU, O. - ALLARD, V. - MARTRE, P. - SNAPE, J.W. - HEUMEZ, E. - LEGOUIS, J. - MOREAU, D. - BOGARD, M. - GRIFFITHS, S. - ORFORD, S. HUBBART, S. - FOULKES, M.J. 2011. Identification of traits to improve the nitrogen-use efficiency of wheat genotypes. In Field Crops Research, vol. 123, pp. 139-152. DOI:10.1016/j.fcr.2011.05.010.

GUARDA, G. - PADOVAN, S. - DElogU, G. 2004. Grain yield, nitrogen-use eficiency and baking quality of old and modern Italian bread-wheat cultivars grown at different nitrogen levels. In European Journal of Agronomy, vol. 21, pp. 181-192. DOI:10.1016/j.eja.2003.08.001.

HIREL, B. - LEMAIRE, G. 2006. From agronomy and ecophysiology to molecular genetics for improving nitrogen use efficiency in crops. In Journal of Crop Improvement, vol. 15, pp. 369-420. DOI: 10.1300/ J411v15n02_07.

KONVALINA, P. - STEHNO, Z. - MOUDRÝ, J. 2009. The critical point of conventionally bred soft wheat varieties in organic farming systems. In Agronomy Research, vol. 7, no. 2, pp. 801-810.

LeGouis, J. - BEGHIN, D. - HEUMEZ, E. - PLUCHARD, P. 2002. Diallel analysis of winter wheat at two nitrogen levels. In Crop Science, vol. 42, pp. 1129-1134.

MOLL, R.H. - KAMPRATH, E.J. - JACKSON, W.A. 1982. Analysis and interpretation of factors which contribute to efficiency of nitrogen utilization. In Agronomy Journal, vol. 74, pp. 562-564.

ORTIZ-MONASTERIO, J.I.R. - SAYRE, K.D. - RAJARAM, S. - McMAHON, M. 1997. Genetic progress in wheat yield and nitrogen use efficiency under four nitrogen rates. In Crop Science, vol. 37, pp. 898-904.

RAUN, W.R. - JOHNSON, G.V. 1999. Improving nitrogen use efficiency for cereal production. In Agronomy Journal, vol. 91, pp. 357-363.

UZIK, M. - ZOFAJOVA, A. - BOLVANSKY, M. 2012 b. Relative nitrogen translocation and accumulation in grain of winter wheat cultivars. In Cereal Research Communications, vol. 40, pp. 285-295. DOI: 10.1556/CRC.40.2012.2.13.

UZIK, M. - ZOFAJOVA, A. 2007. Translocation of dry matter in ten winter wheat cultivars released in the years 1921-2003. In Cereal Research Communications, vol. 35, pp. 1583-1592. DOI: 10.1556/ CRC.35.2007.4.5.

UZIK, M. - ZOFAJOVA, A. 2009. Response of winter wheat cultivars to $\mathrm{N}$ fertilization in grain yield and its components. In Agriculture (Pol'nohospodárstvo) vol. 55 , pp. 171-182.

UZIK, M. - ZOFAJOVA, A. 2012a. Progress in accumulation and translocation of nitrogen in winter wheat cultivars released in the years 1921-2003. In Cereal Research Communications, vol. 40, pp. 135-146. DOI: 10.1556/CRC.40.2012.1.15.

WANG, R.F. - AN, D.G. - HU, C.S. - LI, L.H. - ZHANG, Y.M. - JIA, Y.G. -TONG, Y.P. 2011. Relationship between nitrogen uptake and use efficiency of winter wheat grown in the North China Plain. In Crop \& Pasture Science, vol. 62, pp. 504-514. http://dx.doi. org/10.1071/CP10383.

Received: July 3, 2013 